\title{
Chronic Pancreatitis and Hypoalbuminemia in a Case of End-Stage Renal Disease for Renal Transplantation
}

\author{
Authors \\ Rohan Philip Varghese ${ }^{1}$, Deepak Kumar Chitralli ${ }^{2}$ \\ Department of Nephrology, Columbia Asia Referral Hospital, Bengaluru, India \\ Email: rohanphilipv@gmail.com \\ drchitralli@gmail.com
}

\section{Abstract}

Objective: This report is to show the association of Hypoalbuminemia, pre-and post-transplant, with Acute Graft Kidney Rejection.

Case: This is a case report of a 42-year-old Caucasian male with a history of Type II Diabetes, Hypertension and End Stage Renal Disease on Maintenance Haemodialysis, who was an elective for Renal Transplantation, presented with persistent diarrhoea and marked low albumin levels.

Conclusion: Low albumin levels are a useful auxiliary for assessment of renal graft rejection, but not a prognostic indicator for the same.

Keywords: Hypoalbuminemia, Renal Transplantation, acute rejection, graft failure.

\section{Case}

A 42-year-old male, a known Diabetic and Hypertensive on medical management, started developing facial puffiness and swelling of both lower limbs in the past 2 years. He was diagnosed to have Chronic Kidney Disease (CKD) with a Serum Creatinine value of $4 \mathrm{mg} / \mathrm{dl}$. He was treated conservatively with Nephro-protective agents, however his renal functions steadily deteriorated over the next 2 years to attain End Stage Renal Disease (ESRD). In view of ESRD, raised Creatinine $(5.7 \mathrm{mg} / \mathrm{dl})$, and advanced uremic symptoms, he was initiated on Haemodialysis after Right IJV/CVC (internal jugular vein/central venous line) catheterisation.

He was planned for Live Related Donor Renal Transplantation, with voluntary renal donor being his mother. Assessment for Donor \& Recipient was undertaken by the Cardiologist, Pulmonologist, Gastroenterologist, and Psychiatrist for work-up towards the transplant. Evaluation of Recipient:

1. Known case of Type II Diabetes Mellitus in the past 22 years - On medical management with Insulin (Human Mixtard). $\mathrm{He}$ is irregular with treatment and follow-up visits. Disease has progressed to result in Diabetic Retinopathy and Nephropathy.

2. Gives a history of Diarrhoea since $>22$ years - Large in volume, watery \& oily in consistency, 6-7 episodes/day, non-foul smelling, not associated with blood, and increased following intake of spicy/oily foods. 
3. Habits - Tobacco consumption (Ghutka) and Alcohol since 15 years \& stopped 3 months prior to initiation of dialysis.

Following management of co-morbidities \& deranged blood values, the recipient was evaluated as fit for the renal transplant procedure. He was initiated on Triple Immunosuppressive therapy 2 days prior to the surgery (Tacrolimus, Mycophenolate Mofetil, and Prednisolone).

The procedure was uneventful devoid of postoperative complications.

In the post-operative period, Serum Creatinine levels worsened, persistently low albumin levels were noted, and the Doppler study showed signs s/o acute rejection. An underlying infection was appropriately managed, and pulse steroid therapy was given with regards to graft rejection. Following which, Serum Creatinine levels had gradual improvement, and repeat Doppler studies showed recovery.

Serum Albumin levels recovered gradually as well with Albumin infusions and dietary changes. Simultaneous improvement in generalized oedema was noted, with subsequent weight decrease.

Post-discharge, the recipient is on regular followup as mandated for post-renal transplant patients, and noted to be in good condition with blood parameters within normal limits.

\section{Investigations}

\section{Pre-transplant}

1. Endoscopy and Colonoscopy were inconclusive. Colonoscopy biopsy revealed features of Microscopic Colitis.

2. CT oncology was performed - revealed: Complete Atrophy/fatty replacement of pancreas. An impression of Chronic Pancreatitis with exocrine and endocrine insufficiency was made.

3. Blood tests revealed Hypoproteinemia and Hypoalbuminemia

4. Blood groups of Donor and Recipient were confirmed to be ' $\mathrm{O}$ ' positive.
5. Cross-matching: Complement-dependent cytotoxicity (CDC) cross-match and Donor Specific Antibody (DSA) tests were negative.

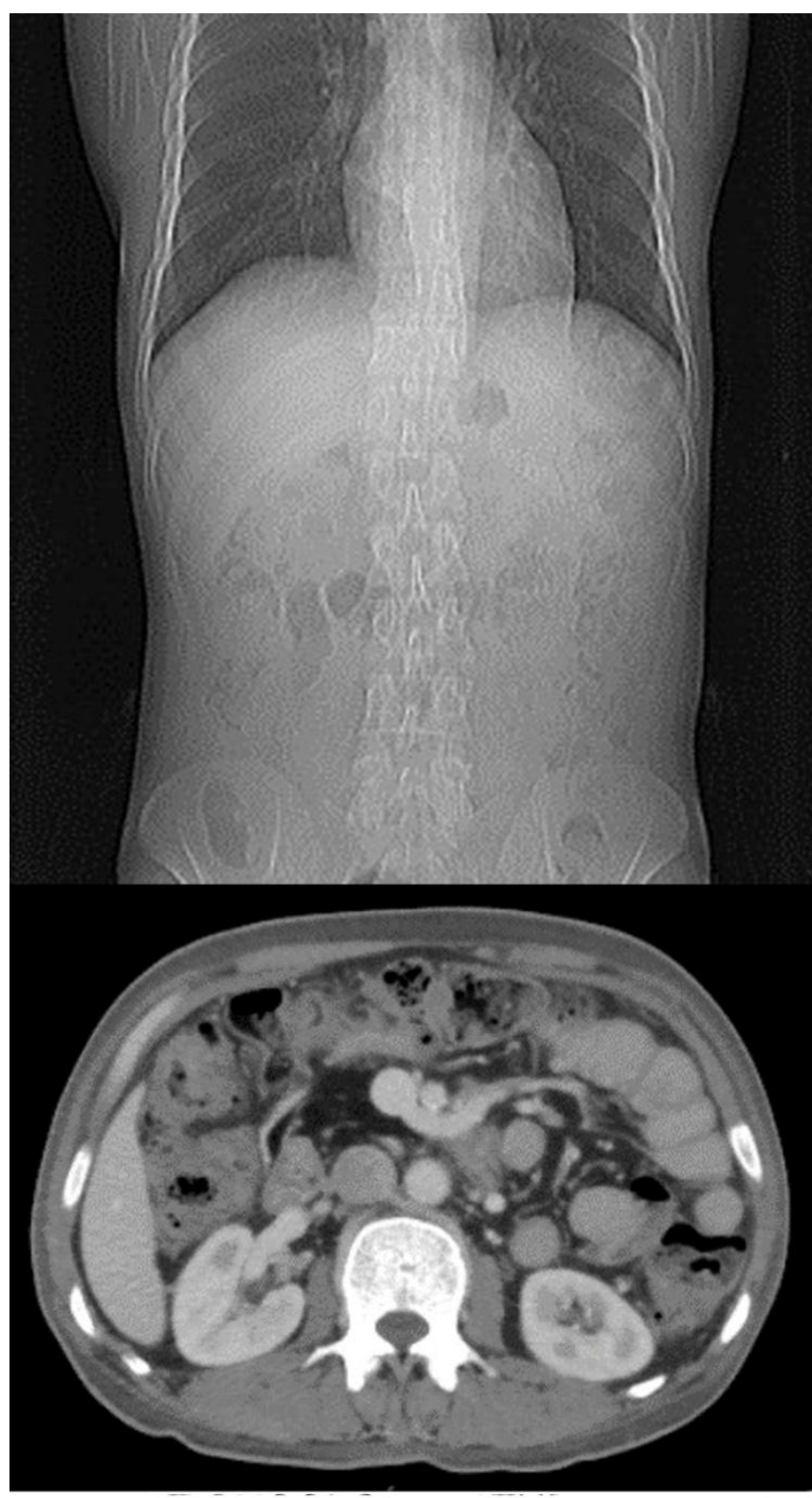

CT ONCOLOGY STUDY: COMPLETE FAT REPLACEMENT/ATROPHY OF PANCREAS

\section{Post-transplant}

1. Doppler of the Renal graft showed uniform perfusion with an RI of 0.7 (Fig. a). From POD (post-operative day) 2, marked generalized oedema and a 10-litre fluid retention was noted (increase in weight from $48 \mathrm{~kg}$ to $58 \mathrm{~kg}$ ). 
2. Serum Albumin levels showed marked Hypoalbuminemia post-procedure (1.4 $\mathrm{mg} / \mathrm{dl})$.

3. Repeat Renal Function Tests (RFT's) showed worsening Creatinine levels, and a further Doppler study showed absent diastolic flow in MRA with reversals in smaller vessels s/o acute rejection (Fig. b). Total Leukocyte counts were markedly raised (19,000 cells/cumm),

4. Creatinine levels continued to deteriorate $(1.58 \mathrm{mg} / \mathrm{dL} \rightarrow 1.93 \mathrm{mg} / \mathrm{dL})$,

5. After appropriate management, Creatinine levels trended downwards $(1.93 \mathrm{mg} / \mathrm{dL} \rightarrow 1.52 \mathrm{mg} / \mathrm{dL} \rightarrow 1.08 \mathrm{mg} / \mathrm{dL})$, and (Fig. c) Doppler graft showed marked improvement.

6. At discharge time, Serum Albumin level was noted to be $3.5 \mathrm{~g} / \mathrm{dL}$.

\section{Treatment}

1. Following Endocrinology opinion, blood sugars were monitored and Insulin dose was corrected according to sliding scale.

2. Diarrhoea was attributed to be caused by Colitis and Pancreatic insufficiency. Hence, he was commenced on pancreatic enzyme supplementation (Pancreatin 25,000U), oral proton pump inhibitors (PPI's), and appropriate dietary changes (avoiding oily and spicy foods).

3. In view of low albumin levels pretransplant, Human Albumin 20\% infusion was instilled. High protein diet was also advised, and followed.

4. In view of persistent Hypoalbuminemia post-transplant, continuous Human Albumin 20\% infusion was commenced, with regular monitoring of albumin levels.

5. Tacrolimus levels were monitored and maintained between $5-10 \mathrm{ng} / \mathrm{mL}$, with the dose adjusted according to body weight.

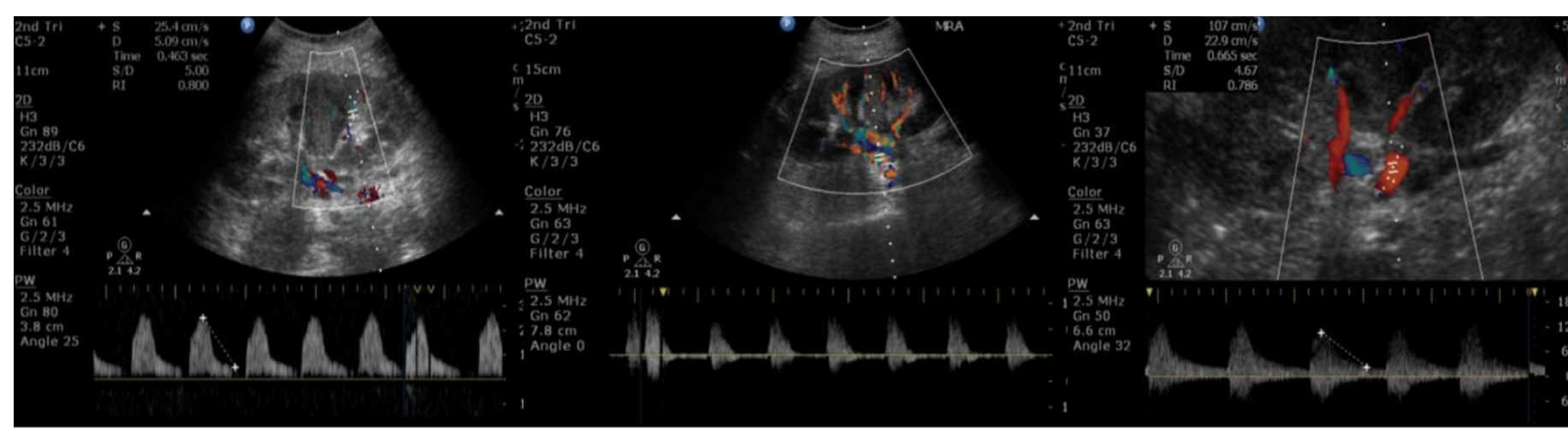

DOPPLER STUDY: a) NORMAL STUDY WITH UNIFORM PERFUSION AND RI - 0.7; b) ABSENT DIASTOLIC FLOW WITH REVERSAL OF FLOW NOTED;

\section{Discussion}

Hypoalbuminemia refers to a state where the Serum Albumin levels in the body are abnormally low. Albumin is synthesized in the liver, and low serum albumin levels serve as an indicator of liver failure, cirrhosis, chronic hepatitis, chronic malnutrition and even as a part of protein losing enteropathies like nephrotic syndrome. ${ }^{[1]}$ Normal serum albumin levels in healthy adults is approximately 3.5 to $5.0 \mathrm{~g} / \mathrm{dL}$. Levels below 3.5 $\mathrm{mg} / \mathrm{dL}$ indicate Hypoalbuminemia.
In the progress of several diseases, low albumin levels have been seen associated with cancer, infection, and inflammation. In our patient, End stage Renal Disease, Chronic Pancreatic Insufficiency and poor dietary intake combined may have contributed to Hypoalbuminemia. This mechanism is not defined; however, it could be as a result of an inflammation response regulated by IL-6 and other inflammatory cytokines. ${ }^{[2]}$ Other proposed mechanisms include reduced nutrition which in turn reduces the ability of the liver to synthesis albumin. It could also be attributed to 
increased vasopermeability secondary to stress response, augmenting albumin entry into tissue spaces. ${ }^{[3]}$

In association with transplantation, Serum albumin levels prior to the procedure were inversely associated with graft failure. In a Cox proportional model, each $1 \mathrm{~g} / \mathrm{dL}$ increase was associated with a $20 \%$ reduction in the risk for graft failure over an average follow-up of approximately 4 years. ${ }^{[4]}$ Very low and low serum albumin (2.50-3.49 g/dL) levels were associated with a higher risk for graft failure compared to patients with serum albumin levels of at least 3.50 $\mathrm{g} / \mathrm{dL}$.

\begin{tabular}{|l|c|}
\hline \multicolumn{2}{|l|}{ HYPOALBUMINEMIA TREND (in this patient) } \\
\hline DATE & SERUM ALBUMIN LEVELS (g/dL) \\
\hline $08 / 02 / 2018$ & 1.7 \\
\hline $02 / 04 / 2018$ & 1.5 \\
\hline \multicolumn{2}{|l|}{ Date of Renal Transplant $-\mathbf{1 9 / 0 4 / 2 0 1 8}$} \\
\hline $20 / 04 / 2018$ & 1.5 \\
\hline $24 / 04 / 2018$ & 1.4 \\
\hline $27 / 04 / 2018$ & 2.5 \\
\hline $28 / 04 / 2018$ & 2.9 \\
\hline $02 / 05 / 2018$ & 2.8 \\
\hline $05 / 05 / 2018$ & 3.1 \\
\hline $19 / 05 / 2018$ & 3.5 \\
\hline
\end{tabular}

In context, a retrospective study by Moore et al, analysed data from 2763 adult kidney transplant recipients enrolled in a long-term study, where multiple regression analysis revealed that Hypoalbuminemia in the 6 months prior to the transplant, was independently associated with renal transplant failure. ${ }^{[5]}$ Yang et al also found a causal relationship between serum albumin level and renal graft failure. In their study, the relative risk of graft failure was lowest in the group with high serum albumin levels pre-transplant. Chronic rejection and delayed graft function were of increased incidence in patients with pre-transplant Hypoalbuminemia. $^{[6]}$ These authors concluded that Hypoalbuminemia before kidney transplantation is associated with more serious complications and worse short- and long-term graft outcomes.

In our research study, all variables that could cause rejection were ruled out: Blood group incompatibility, Tacrolimus levels, compliance, and infection. As previous research indicated the association of low albumin levels with graft rejection, we noted a close relation to the same.

However, the predictive function of albumin levels is not reliable: a) In the pre- and posttransplant period, infusion of human albumin was done, which could lead to error in our research. b) Intra-individual variations were not covered due to limited sample pool. ${ }^{[3]}$

\section{Conclusions}

In conclusion, our present study shows the effect of Low albumin levels on acute graft rejection. We suggest that Albumin levels could be a valuable tool in assessment of rejection, but should not be considered as an indicator.

\section{Acknowledgements}

We are grateful to Columbia Asia Hospital, Yeshwantpur, Bangalore and the Department of Nephrology and Renal Transplant Surgery.

Funding: The study did not get any funding.

\section{Availability of data and Materials}

No additional data is available, in view of maintaining doctor-patient confidentiality and any further divulge in information may prove detrimental to that clause.

\section{References}

1. Caraceni P, Tufoni M, Bonavita ME. Clinical use of albumin. Blood Transfus. 2013;11 Suppl 4(Suppl 4):s18-25.Li, S., Zhang, Y., Li, M., Xie, C., \& Wu, H. (2017). Serum albumin, a good indicator of persistent organ failure in acutepancreatitis. BMC gastroenterology, 17(1),59.doi:10.1186/s12876-017-0615-8

2. Tanaka T, Narazaki M, Kishimoto T. IL-6 in inflammation, immunity, and disease. Cold Spring HarbPerspect Biol. 2014;6(10):a016295 Published. doi:10.1101/cshperspect.a016295

3. Li S, Zhang Y, Li M, Xie C, Wu H. Serum albumin, a good indicator of persistent 
organ failure in acute pancreatitis. $B M C$ Gastroenterol. 2017;17(1):59. Published 2017 Apr 26. doi:10.1186/s12876-0170615-8

4. Molnar MZ, Kovesdy CP, Bunnapradist S, et al. Associations of pretransplant serum albumin with post-transplant outcomes in kidney transplant recipients. Am J Transplant. 2011;11(5):1006-15.

5. Moore, Jason \& He, Xiang \& Shabir, Shazia \& Hanvesakul, Rajesh \& Benavente, David \&Cockwell, Paul \& Little, Mark \& Ball, Simon \&Inston, Nicholas \& Johnston, Atholl\& Borrows, Richard. (2011). Development and Evaluation of a Composite Risk Score toPredict Kidney Transplant Failure. American journal of kidney diseases : the official journal of the National Kidney Foundation. $\quad 57 . \quad 744-51$. 10.1053/j.ajkd.2010.12.017.

6. Yang SW, Choi JY, Kwon OJ. The impact of pretransplantation serum albumin levels on long-term renal graft outcomes. Transplant Proc. 2013;45:1379-138. 